\title{
La mosaïque culturelle et babélienne de Panero
}

Polymorphie et polysémie d'une poétique critique du renouveau

\section{Marjolaine Piccone-Miloud}

\section{(2) OpenEdition \\ Journals}

Édition électronique

URL : http://journals.openedition.org/agedor/713

DOI : 10.4000 /agedor.713

ISSN : 2104-3353

Éditeur

Laboratoire LISAA

Référence électronique

Marjolaine Piccone-Miloud, "La mosaïque culturelle et babélienne de Panero », L'Âge d'or [En ligne],

7 | 2014, mis en ligne le 01 mars 2014, consulté le 19 avril 2019. URL : http://journals.openedition.org/ agedor/713 ; DOI : 10.4000/agedor.713 


\title{
La mosaïque culturelle et babélienne de Panero : polymorphie et polysémie d'une poétique critique du renouveau
}

\begin{abstract}
Résumé : En s'inscrivant contre la norme et l'Idéal, Panero entend faire imploser l'espace sémantique et morphologique du poème. Toutefois, cet anéantissement se veut paradoxalement bien plus fécond qu'il n'y paraît et tient moins, au final, de la liquidation que d'un débordement. En effet, le poète élabore un nouveau système référentiel qui orchestre une véritable symphonie idiomatique et culturelle dans son œuvre. En entremêlant les références les plus hétérogènes, des plus érudites aux plus populaires, Panero crée un heurt référentiel et intertextuel, une saturation sémantique. Le discours poétique se meut en une anormalité culturelle dont l'extraordinaire ampleur le métamorphose à la fois en passeur de cultures et en médiateur des arts. Si démantèlement du poème traditionnel il $\mathrm{y}$ a, il participe d'un renouveau indubitable. Celui-ci s'articule autour d'une expansion du poème, par le biais de celle du langage et de la signification qui, entrant en résonance avec un réseau culturel sans limite, se transcendent à l'infini. Ainsi, cette énergie créatrice du chaos que libère le poète démiurge, catalyse en réalité un passage vers une forme d'universalité, dont le lecteur est appelé à devenir un acteur essentiel.
\end{abstract}

Mots-clés : Intertextualité - Référentiel - Arts- Babel - Polysémie - Polyphonie- Culturalismo

Resumen: Por inscribirse contra el Ideal y la norma, Panero pretende hacer implosionar el espacio semántico y morfológico del poema. Sin embargo, este aniquilamiento se pretende paradójicamente mucho más fecundo que lo parece y, finalmente, se parece más a un desbordamiento que a una liquidación. En efecto, elabora el poeta un nuevo sistema referencial que orquesta una verdadera sinfonía idiomática y cultural en su obra. Por entremezclar las referencias más heterogéneas, de las más eruditas hasta las más populares, Panero crea una colisión referencial e intertextual, una saturación semántica. El discurso poético se transforma en una anormalidad cultural cuya extraordinaria magnitud lo metamorfosea a la vez como un puente entre culturas y mediador de las artes. Si se trata del desmantelamiento del poema tradicional, tiene elementos de una indudable renovación. Dicha renovación se articula en torno a una expansión del poema, mediante la del lenguaje y la de la significación que, por entrar en resonancia con una red cultural sin límites, se trascienden hasta el infinito. Así, esta energía creadora del caos que desencadena el poeta demiurgo, cataliza en realidad un paso hacia una forma de universalidad, de la cual está llamado el lector a volverse un actor esencial.

Palabras clave: Intertextualidad - Mosaico - Artes - Babel -Polisemia - Polifonía - Culturalismo

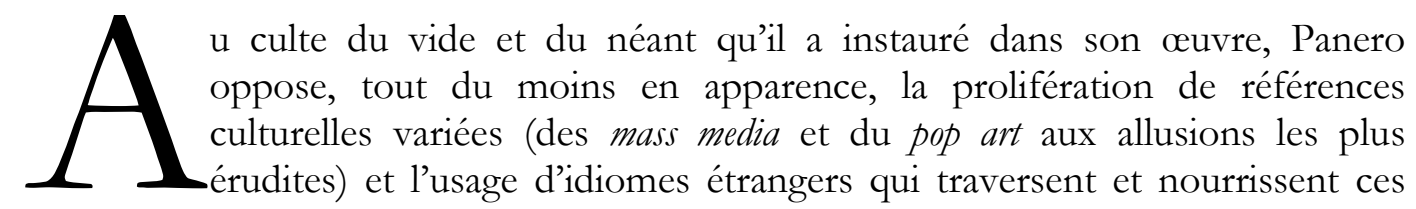


poèmes. Or, cette abondance ambiguë n'est en réalité qu'un autre aspect du processus de «liquidation» et de provocation panérien. Poésie du dérèglement, de l'étrangeté, de la disjonction, l'œuvre de Panero se radicalise dans la polyphonie et l'intertextualité. Elle tronque les attentes, multiplie les niveaux de lecture, entrave la compréhension, se joue des repères du public, transgresse les topoï et réinvente une tradition littéraire, mais aussi provocatrice.

En détournant et en unissant les arts, les langues, les idéologies et les sens (dans la double acception des cinq sens et des significations), Panero déploie une veine critique fondée sur de nouvelles alliances formelles et sémantiques fécondes. Il façonne alors un nouveau lecteur, forgé par une culture composite, voire disparate, et apte à relever ce défi herméneutique. Cependant, par cette insatiable transcendance du poème et du langage, il semble que Panero tende paradoxalement vers une forme d'universalité hors-normes, médiatrice des cultures. Ainsi, comment cette mosaïque culturelle et babélienne participe-telle finalement d'une double dynamique critique de destruction et de renouveau ?

\section{L'entremêlement des cultures comme ferment de subversion}

De fait, cet entremêlement des cultures - en dépit des apparences qui jouent contre Panero - fait l'objet d'une construction remarquable de méticulosité et de rigueur. En effet, les références ne sont jamais l'objet du hasard mais interviennent au contraire toujours dans un circuit réfléchi dans l'optique de désorienter le lecteur. Ferment de subversion évident, ce canevas extrêmement dense participe à la fois d'un discours méta-poétique critique et d'une dynamique indéniable de renouveau. Le poème se présente sous des traits inédits où la concentration des références culturelles le nourrit autant qu'elle l'anéantit par le poids des éléments qui s'amoncellent.

Tout d'abord, le poète, persistant dans sa critique de la norme, entend multiplier les tensions formelles et thématiques au sein de chaque poème. Par l'entremise de ces références culturelles tout à fait hétérogènes, il met donc en scène une première didactique : le duel du savant et du populaire. Peut-être est-il intéressant de revenir prudemment sur la notion de culturalismo que Guillermo Carnero, appartenant également à la génération polémique des Novísimos, a mise en lumière. Mode d'écriture traversant les recueils, et bien plus phénomène provocateur, il renvoie directement à la vive critique novísima de la poésie sociale de l'après-guerre. Dans l'optique de redonner à la poésie ses lettres de noblesse, il consiste d'une part à enrichir le discours par des motifs culturels extérieurs, plus ou moins évidents à déceler car plus ou moins érudits, ou plus ou moins dissimulés. D'autre part, il se définit par l'usage ponctuel d'un lexique spécifique, parfois technique, parfois suranné, souvent complexe (à la manière d'un Huysmans dans $A$ rebours par exemple, quoique de façon nettement plus extrémiste). Cependant, il est nécessaire de retenir que cette dynamique n'a rien d'une démarche élitiste. Transgressive peut-être, mais il n'est nullement question de rendre inaccessible ou hermétique la poésie. Il s'agit bien plus de considérer ce choix comme un moyen de nourrir le texte et pour que l'accès au sens se mérite. Un lien de connivence nait alors entre le poète et son lecteur, autour d'un patrimoine commun et du partage d'une poésie fascinante qui se dérobe ou se révèle, par touches. Songeons par exemple au poème "Figuras de la pasión del Señor », où les derniers vers assimilent Dieu à une victime de Jack l'Éventreur : 


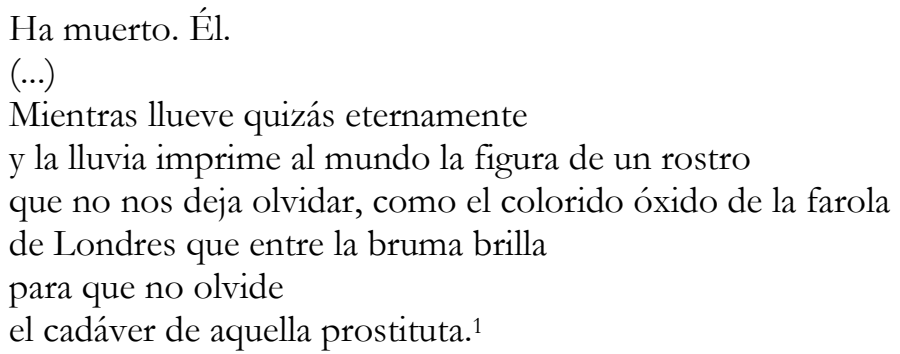

Ce jeu avec des références extérieures à la poésie, moins érudites que populaires, provoque des failles dans la tradition : Panero se plaît à faire cohabiter des référents antithétiques - ici profanes et religieux - qui deviennent alors blasphématoires par leur hétérogénéité. Songeons encore au poème «Blancanieves se despide de los siete enanos » qui s'ouvre sur la référence au conte de fée - très vite pervertie - et qui s'achève sur une mention à $L a$ Cerisaie de Tchekov, qui est à vendre. Ainsi, le titre sous-entend un développement référentiel autour d'un conte populaire, tandis que la clausule éteint le poème sur une référence plus savante à la littérature russe :

Prometo escribiros, pañuelos que se pierden en el horizonte, risas que palidecen, rostros que caen sin peso sobre la hierba húmeda, donde las arañas tejen ahora sus azules telas. En la casa del bosque crujen, de noche, las viejas maderas, el viento agita raídos cortinajes, entra solo la luna a través de las grietas. Los espejos silenciosos, ahora, qué grotescos, envenenados peines, manzanas, maleficios, qué olor a cerrado, ahora, qué grotescos. Os echaré de menos, nunca os olvidaré. Pañuelos que se pierden en el horizonte. A lo lejos se oyen golpes secos, uno tras otro los árboles se derrumban. Está en venta el jardín de los cerezos. ${ }^{2}$

Pour mieux déjouer les attentes et intensifier les décalages - pour le moins brutaux - au sein de son œuvre, Panero travaille à la constante cohabitation de références érudites pointues et de références plus communes. Aux poèmes en apparence (et nous insistons sur l'expression "en apparence ») axés sur l'univers des contes de fées ou des mass medias, tels «El estreno en Londres de 'Mary Poppins' ", "The universal soldier »", "Oh Flash Gordon $»^{5}$, se heurtent des poèmes comme "Le châtiment de Tartuffe $»^{6}$ en français dans le texte qui fait écho à un poème éponyme de Rimbaud, ou "Le marchand d'ail et d'oignon $\gg^{7}$ qui, lui, renvoie à un poème de Mallarmé. Et les exemples affluent sans être aussi extrêmes : pensons à " 20000 leguas de viaje submarino » ${ }^{8}$ ou « La fábula de la cigarra y la hormiga ${ }^{9}$ dont les références, quoique littéraires, à Jules Verne et La Fontaine restent toutefois plus accessibles que «Boris Pasternak ¿Eres tú el que lloras? " ${ }^{10}$. Nous n'évoquons ici que les titres de poèmes, mais ce duel ambivalent se poursuit et s'intensifie à l'intérieur du corps poèmatique, jusque dans ses recueils les plus récents. Il y a, par conséquent, interférence constante entre ces deux domaines de connaissances et, de ce choc perpétuel, naît une forme de perversion et de dérision à l'égard des références

\footnotetext{
${ }^{1}$ PANERO, Leopoldo María, Poesía completa 1970-2000, Madrid, Visor Libros, 2006, p. 387.

2 PANERO, Leopoldo María, Poesía completa 1970-2000, op. cit., p. 62.

3 Op. cit., p. 36.

${ }^{4}$ Op. cit., p. 46.

5 Op. cit., p. 55.

${ }^{6}$ Op. cit., p. 79.

7 Op. cit., p. 421.

8 Op. cit., p. 68.

9 Op. cit., p. 441.

10 Op. cit., p. 497.
} 
culturelles. Au final Panero déconstruit son ouvrage à mesure qu'il l'édifie. L'abondance de motifs aussi divergents et aussi nombreux semble servir la liquidation panérienne. L'excès intertextuel ou plus largement référentiel apporte dans un premier temps un nouveau souffle poétique qui, très vite, se révèle toxique et annihile la parole poétique. Celle-ci est alors noyée sous le charriage de matériaux hétéroclites qui corrompent le poème.

Par ailleurs, tiraillé entre la nécessité d'un renouveau et l'impossible fusion des cultures, Panero envisage une solution ambiguë : le topos. Entre stérilité et transfiguration, le lieu commun, à propos duquel Baudelaire disait "Créer un poncif, c'est le génie »", s'impose comme un pont reliant la culture savante et la culture populaire. Nous en avons un exemple flagrant dans le poème « La matanza del día de San Valentín »:

King-Kong, asesinado. Como Zapata. ¿Por qué no, Maiacovsky? O incluso Pavese. La maldición. La noche de tormenta. Dies irae. La mentira de Goethe antes de morir. Las treinta monedas. La sombra del patíbulo. Marina Cvetaeva, tu epitafio serán las inmensas praderas cubiertas de nieve. ${ }^{12}$

Concentré de références, ce court poème haché accumule et oppose les connaissances. L'ouverture apoétique sur "King-Kong », emblème du cinéma de masse, laisse présager d'un poème focalisé sur l'univers enfantin auquel Panero est indéfectiblement attaché. Pourtant, dès le segment suivant, l'hypothèse est tronquée par les cinq figures qui se succèdent: Zapata, leader de la révolution mexicaine, Maïakovski, poète et dramaturge russe, Pavese, poète italien, Goethe qu'on ne présente plus, et Marina Cvetaeva, poétesse russe. Une nouvelle fois le lecteur est confronté à l'imbrication de références aussi évidentes qu'ardues. Sans compter la dissimulation au milieu de cette galerie de portraits, de la mention implicite aux «trente pièces d'argent », allusion à la somme remise à Judas pour avoir livré Jésus. Or, c'est précisément ce segment qui doit nous alerter: Panero ne se contente pas de mettre bout à bout des éléments féconds, il joue sur leur nature topique. En effet, ces trente pièces d'argent font partie des lieux communs du christianisme, des épisodes qui relèvent bien plus de la culture générale que de la culture religieuse. Ainsi, Panero s'emploie à désamorcer, à démythifier les références érudites pour davantage homogénéiser le poème et le rendre accessible, précisément par sa nature insensée. Alors même qu'on l'aurait sanctionné presque d'incohérence profonde - ce qui eût été commettre une erreur profondément réductrice - apparaît que l'enjeu du poème n'est pas le déchiffrement intégral de son sens, mais l'acceptation de son contre-sens.

Rien (ou presque) n'est hasardeux chez Panero, chaque référence fait partie d'un système. Et le cliché est la clef de voûte de cet ensemble qu'il soutient autant qu'il le rend caduc puisqu'il en trahit l'inconséquence, il en dénonce la facticité. Pensons notamment à

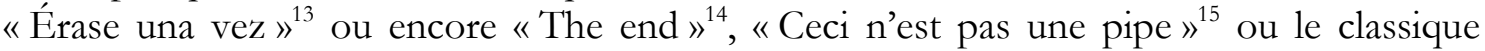
«Hymne à Satan ${ }^{16}$, ressort blasphématoire désormais vidé de sa substance subversive par des années de surconsommation. La plupart des titres sont des lieux communs qui, au final, n'ont rien à voir avec le contenu des poèmes : ils ont vocation à semer le trouble et à déjouer les attentes du public. Citons par exemple «Go down Moses »" «La huida a

\footnotetext{
11 BAUDELAIRE, Charles, CEuvres Complètes II, Paris, Gallimard, Bibliothèque de la Pléiade, 1976, p. 662.

12 PANERO, Leopoldo María, Poesía completa 1970-2000, op. cit., p. 35.

13 Op. cit., p. 67.

14 Op. cit., p. 262.

15 Op. cit., p. 481.

16 Op. cit., p. 364 et 467.

17 Op. cit., p. 34.
} 
Egipto ${ }^{18}$ ou «El retorno del hijo pródigo » qui semblent inaugurer une référence religieuse alors qu'il n'en est rien :

\begin{abstract}
¿No ha mirado Vd. nunca dentro del teléfono? Él sí lo hizo, y se dio cuenta de que al otro lado estaban las dos latas atadas por un hilo en Juegos y Pasamientos del Tesoro de la Juventud. Sí, las latas y el hilo de cobre, se introdujo en el auricular como en un portal oscuro, llegó a su casa, algo tarde para merendar. ${ }^{19}$
\end{abstract}

Panero se plaît à tromper son lecteur et à dynamiter les carcans culturels. Il s'agit bien moins de blasphémer que de provoquer en détournant les références. Le poète manifeste une volonté de rompre avec les normes établies. Et à la sclérose habituelle que provoque l'emploi du poncif, succède une forme de rénovation puisqu'il est lui-même instrumentalisé et fait l'objet d'une transcendance polysémique.

Autre aspect majeur de ce choc des cultures révolutionnaire, le bal des idiomes que donne Panero. Défi babélien, il occasionne une refonte complète de la parole poétique, transfigurée par la rénovation en profondeur du langage par l'interaction d'autres langues. Le locuteur se " vaporise $»^{20}$ dans l'entremêlement des langues pour devenir porte-parole du discours, porte-parole du poète, porte-parole du poème. Meschonnic parle d'une «disparition élocutoire du sujet (...) La modernité est une fonction du langage, du discours $»^{21}$. Le «je» a désormais un statut complexe, contradictoire et plural. La dissolution du sujet le rend particulièrement ambigu. Il oscille entre abstraction et concrétisation. De fait, dans la mosaïque idiomatique, le sujet s'anamorphose. Si bien que ce que nous prenions pour une réduction ontologique s'avère être, en réalité, l'exact opposé : une expansion. En s'éloignant de toute représentation identitaire, il atteint une autre dimension. Perpétuellement transfiguré, le sujet polyglotte est une antithèse insoluble qui laisse la priorité à un langage universel.

Les multiples incursions du français, de l'anglais et de l'italien (voire plus rarement de l'allemand, du latin, du grec et du russe) alimentent l'élan corrupteur et destructeur du poème, et, simultanément, offrent un nouvel horizon à la parole poétique, dont l'écho libéré, se propage et tend ainsi à l'universel. Panero mobilise une palette linguistique qui introduit durablement la dissonance dans le poème, une forme de chaos sonore qui engendre logiquement un chaos sémantique. "Le châtiment de Tartuffe ${ }^{22}$ (relevons la faute qui redouble par erreur la consonne « $\mathrm{f} »)$ propose un titre en français et un poème en anglais, son recueil Siete poemas se veut résolument multilingue puisqu'il contient un poème en italien, deux en anglais et quatre en espagnol. Dans l'anthologie à laquelle nous nous référons, Poesía completa (1970-2000), nous décomptons quarante-trois poèmes dont les titres ne sont pas en espagnol, dont une majorité en français. Notons que souvent seuls les titres offrent une vitrine dans une autre langue, ce qui souligne d'autant plus la volonté transgressive de Panero qui entend désarçonner son lecteur dès le premier coup d'œil. À ceci près que la récurrence du français renforce le poids des intertextes de la littérature française qui dominent (à commencer par l'omniprésent Stéphane Mallarmé, Arthur Rimbaud, Charles Baudelaire, François Villon, Jules Verne, La Fontaine, Tristan Corbière -

\footnotetext{
18 Op. cit., p. 53.

${ }^{19}$ Op. cit., p. 41

20 «De la vaporisation et de la centralisation du moi, tout est là ». BAUDELAIRE, Charles, CEuvres Complètes II, op. cit., p. 676.

${ }^{21}$ MESCHONNIC, Henri, Modernité, Modernité, Paris, Gallimard, 1993, p. 33-36.

22 PANERO, Leopoldo María, Poesía completa 1970-2000, op. cit., p. 79.
} 
«Paris $»^{23}-$, Bataille, Alphonse Daudet - «La arlesiana ${ }^{24}-$, Zola - «Acerca del caso Dreyfuss sin Zola o la causalidad $»^{25}-$, et bien d'autres encore).

Il semble que cela ait indubitablement partie liée avec l'héritage de la poésie française de la fin du XIX siècle sur Panero. Les artisans de la Modernité ont profondément marqué la poésie panérienne - en dehors de tout amalgame avec la lignée des poètes maudits - et les empreintes de ces poètes sont nombreuses dans les recueils : Charles Baudelaire y est notamment visible avec le refrain des «Litanies de Satan ${ }^{26}$ repris en exergue de «El himno a Satán $»^{27}$, la série des "Poemas de la vieja $»^{28}$ qui ne sont pas sans rappeler «Les petites vieilles $»^{29} \mathrm{du}$ Français, ou encore «Alba $»^{30}$ pour Rimbaud. Mallarmé relève d'un cas particulier puisqu'il occupe une place croissante dans l'œuvre panérienne : Panero imprime sur ses poèmes la présence du Causeur de la rue de Rome, entrelaçant leurs deux voix au fil des citations, des paratextes, des dédicaces.

Cette surimpression commence à apparaitre dans les années 1990 avec le recueil Contra España y otros poemas no de amor qui contient un premier titre faisant écho au poète: «Lo que Stéphane Mallarmé quiso decir en sus poemas»". Et la tendance va en s'amplifiant: dans Orfebre encore, un titre en français dans le texte reprend cette fois un vers de Mallarmé, «La Destruction fut ma Béatrice ${ }^{32}$; puis deux autres titres en espagnol font écho respectivement au poète et à l'un de ses recueils : «La rosa de Mallarmé »" ${ }^{33}$ et «Un golpe de dados no abolirá el azar» ${ }^{34}$. À cela s'ajouteront «La balada del ahorcado o himno a Mallarmé » ${ }^{35}$ et «Plagiando a Mallarmé ». Notons que la citation capitale pour définir l'art poétique panérien - «La destruction fut ma Béatrice » - ressurgit en maints endroits dans la dernière anthologie de Panero : six occurrences glissées dans le corps du poème et qui vont se rapprochant vers la fin du livre ( $\mathrm{p} 7,56,279,398,537,539,547,584)$. La présence mallarméenne est si forte qu'elle pénètre jusqu'au cœur du poème, Panero déconstruisant, déterritorialisant, son œuvre à l'aide de fragments venus du dehors de l'espace poématique. En attestent notamment deux poèmes de Teoría Lautreamontiana del plagio, "Vejez $»^{37}$ et "Ash Wednesday»" (référence à T.S. Eliot ${ }^{39}$ ) qui contiennent respectivement en leur sein un titre et un vers de Mallarmé : extraits.
Ah, el esclavo de las palabras
el siervo del humo
donde muere todo orgullo
a los pies de la nada

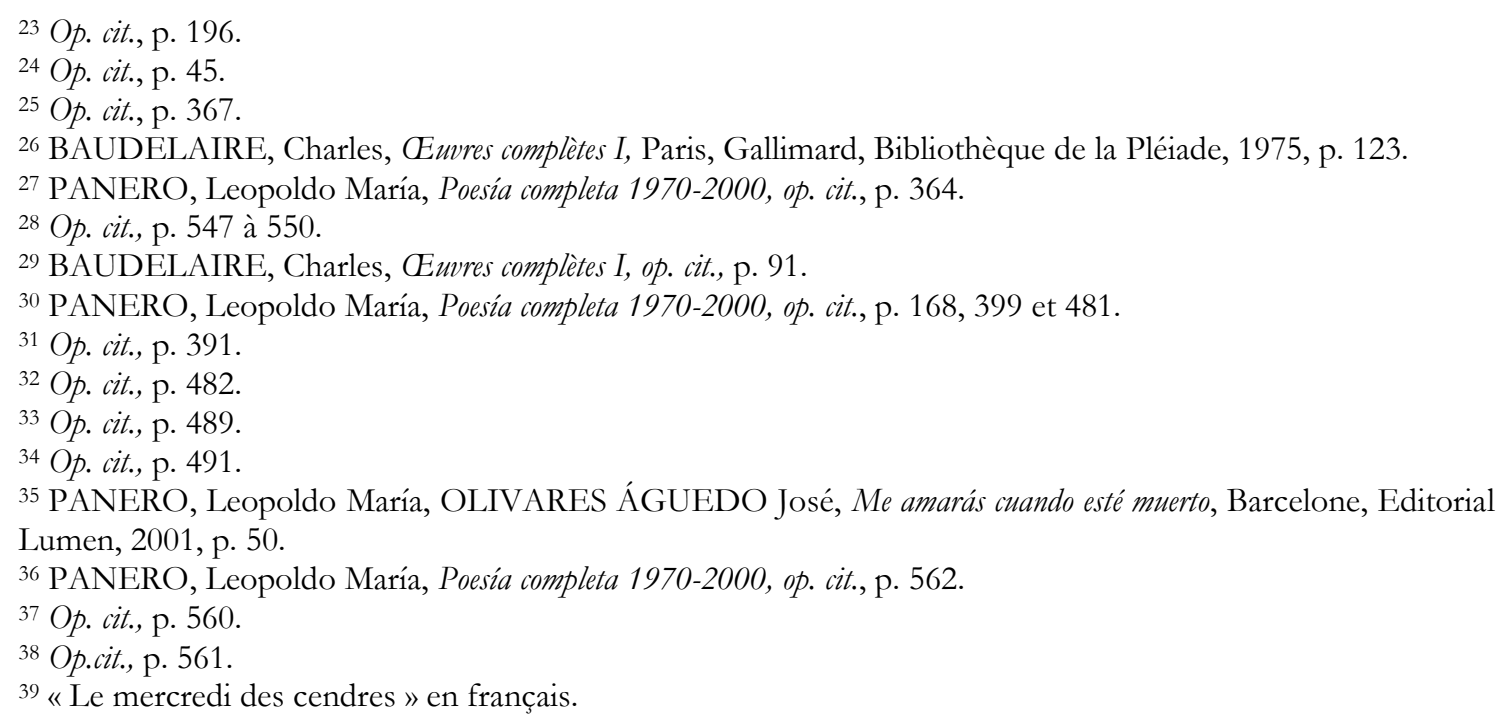


rezándole a la nada

rezando para nada al poema

« tout orgueil fume-t-il du soir $»^{40}$

STÉPHAN MALLARME ${ }^{41}$.

El poema es la casa de la ceniza

(...)

el no-lugar

de donde penden los hombres

que se ahorcan

Ridículamente en el reverbero.

(Mallarmé : « vont se pendre ridiculement au réverbère »"2)

Pourtant, ces références sont rejetées en marge du poème, après la clausule, comme un élément rapporté que le poète prend soin de mettre en lumière sans pour autant le rattacher à son œuvre. La mise en scène est soignée : dans le premier cas, l'emploi des majuscules est trompeur, il attire l'attention mais l'orthographe du nom est volontairement estropiée car Panero n'hispanise pas le prénom comme le souligne l'accent, par conséquent il manque bien le «e » final. Dans le second cas, la mise entre parenthèses, les minuscules et la seule présence du nom propre feignent de minimiser, d'étouffer, la citation alors qu'en réalité, elle est intensifiée par sa traduction littérale en espagnol dans le vers précédent (qui s'ouvre, qui plus est, sur une majuscule qui n'a normalement pas lieu d'être). Elle est ainsi redoublée et, par là, Panero entend mettre à l'index la caducité du vers mallarméen, remplacé par le vers panérien qui relègue son modèle au second plan dans une parenthèse. Au final, il semble que la mention mallarméenne compte d'autant plus qu'elle est dénoncée comme hétéroclite, comme un artifice du poème. De plus, en nommant Mallarmé, Panero joue un double jeu : celui de rendre à la fois explicite et accessible la référence pointue à son lecteur, et de, simultanément, valoriser et banaliser ce marqueur d'érudition. C'est Mallarmé luimême qui s'en trouve démythifié parce que Panero fait voler en éclats le poncif de l'inaccessibilité mallarméenne.

Dans sa dernière anthologie rassemblant les poésies de 2000 à 2010, Panero fond véritablement le discours mallarméen dans le sien, au moyen de citations indirectes et approximatives qu'il attribue nonchalamment au poète français. Il s'agit de prendre en compte la double évolution du système des références paratextuelles et intertextuelles : au croisement de l'intensification des occurrences et de l'affaiblissement de leur impact. En effet, la citation se fait automatisme, mécanisme circulaire bien réglé au cœur duquel vont et viennent régulièrement les fragments littéraires et poétiques d'autrui. Panero pousse même l'exercice à son extrême avec l'auto-citation. Parmi ces références, un peu plus d'une trentaine renvoient précisément à Mallarmé, soit au travers du titre, soit par le biais d'une citation liminaire, soit encore, de manière résumée, au sein même du poème avec les mentions «Mallarmé dixit», «como lo decía Mallarmé» ou bien «comme lo dice Mallarmé ». Ainsi, peut-être se dévoile au travers des références culturelles, une forme

\footnotetext{
40 MALLARMÉ, Stéphane, CEuvres Complètes I, Paris, Gallimard, Bibliothèque de la Pléiade, 1998, p. 41. En français dans le texte.

${ }^{41}$ PANERO, Leopoldo María, Poesía completa 1970-2000, op. cit., p. 560.

42 MALLARMÉ, Stéphane, Euvres Complètes I, op.cit., p. 7. En français dans le texte.
} 
d'hommage rendu, ou, tout du moins, un mouvement cohérent qui se ferait passeur de culture à travers la médiation des arts et des langues.

\section{Quand la déliquescence touche au sublime : focus sur l'héritage radicalisé du dérèglement rimbaldien et mallarméen}

Au paroxysme de la dégradation panérienne, portant aux nues un art certain de l'abîme, affleurent plus que jamais l'héritage rimbaldien, et, en creux, l'influence mallarméenne. L'omniprésence des intertextes ou des paratextes liés à ces deux poètes français de la fin du XIX ${ }^{\mathrm{e}}$ siècle incite à mettre en perspective ces trois œuvres. "Enfer ou Ciel, qu'importe? Au fond de l'inconnu pour trouver du nouveau!» ${ }^{43}$. Ainsi Panero, reprenant le vers évocateur de Mallarmé, "La destruction fut ma Béatrice», se métamorphose en poète démiurge, pour un contre-évangile provocateur et dystopique. À la suite d'Une saison en enfer, il compose une œuvre tout entière vouée à une vie tantôt furieuse tantôt moribonde dans cet univers qu'il investit dans sa totalité. Repensons à l'explicite section de recueil : «Proseguir el infierno » ${ }^{44}$. Ainsi est évoqué le désir d'aller au-delà de ce qui a été fait, de poursuivre un dessein inachevé. Tout comme chez Rimbaud, il est question de recréer un monde, distinct de celui duquel ils se sentent prisonniers. C'est un nouveau royaume des ombres que Panero et Rimbaud forgent, en marge des mythes, des littératures et des religions, du cadastre manichéiste entre le monde des vivants et celui des morts, le paradis des élus et l'enfer des pécheurs. Le mouvement met en scène une chute latente, qui, à défaut d'être brutale, ressemble davantage à un cheminement spirituel sans fin. Panero et Rimbaud composent et décomposent un monde alternatif, une utopie contre la réalité et contre la fiction, contre la norme. Un monde qui s'anamorphose au rythme des illusions et des désenchantements.

Sans revenir sur la figure du poète marginalisé, reste que leurs deux œuvres manifestent une violence de ton envers leurs pays et leurs contemporains. L'hostilité envers l'Espagne se revendique dans plusieurs poèmes, avec pour acmé le recueil Contre l'Espagne et autres poèmes de non amour et son titre sans équivoque. Par ailleurs, dans Last river together, c'est la «Canción del croupier del Mississipi » ${ }^{45}$ qui attire notre attention par la transfiguration du pays en enfer: (...) este lugar parecido al Infierno / que llaman España. Panero et Rimbaud sont les damnés du monde réel. De fait, si l'enfer est traditionnellement lieu d'enfermement et de non-retour, il ne marque pas chez Rimbaud et Panero la fin du voyage. Il ouvre au contraire sur un espace illimité propice à la quête d'une sorte de « contre-Idéal ». Les poètes sont en quête d'un renouveau poétique au travers duquel s'épanouiraient une Autre langue, une Autre beauté. Panero rend ce contre-Idéal possible en un vers du poème "Eve » ${ }^{46}$ qui préfigure déjà le contre-évangile avec une inversion des univers : La Muerte se habia hecho vida ${ }^{47}$. Tout comme Rimbaud qui, dans «Nuit de l'enfer» s'étonne: «Puis-je décrire la vision, l'air de l'enfer (...) Et c'est encore la vie! $»^{48}$. Le poète espagnol désigne régulièrement Satan comme moteur de la création poétique, notamment par le biais de

\footnotetext{
43 BAUDELAIRE, Charles, «Le voyage », Euvres Complètes I, op.cit., p. 134.

${ }^{44}$ PANERO, Leopoldo María, Poesía completa 1970-2000, op. cit., p. 180.

45 Op. cit., p. 219.

46 Op. cit., p. 185.

47 Op. cit., p. 187.

48 RIMBAUD, Arthur, Euvres complètes I, Paris, Gallimard, Bibliothèque de la Pléiade, 2009, p. 255.
} 
l'expression «señor del verso » qui revient dans les poèmes «Himno a Satán » ${ }^{49}$ et dans l'ironique «Oh Señor Jesús $»^{50}$. Ceci se concrétise dans «Al Infierno » ${ }^{51}$ où, comme souvent, le poète et Satan ne font qu'un :

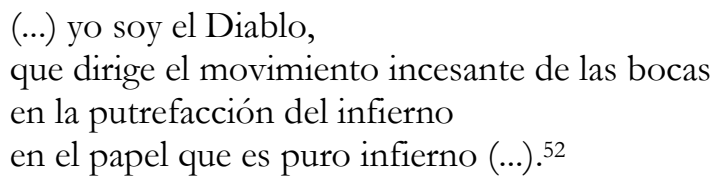

Le locuteur et le diable, unis dans le pronom personnel sujet « je », s'imposent comme les créateurs du poème puisqu'ils sont à l'origine du "mouvement des bouches", "sur le papier ». Et le poème, désigné comme « un pur enfer», psalmodie ce nouvel art poétique du contre-idéal. Parallèlement, s'opère dans leurs œuvres une véritable réécriture biblique qui, en réalité, "désécrit » la Bible pour inventer un Livre inversé où chaque figure est désormais ambivalente, où le manichéisme et la hiérarchie traditionnelle se dissolvent dans un processus de désacralisation. Notons que l'entreprise blasphématoire de Panero est une démarche plus poétique qu'idéologique. Le contre-évangile tente au final d'échapper au tragique de l'existence : qu'il s'agisse de la « réalité rugueuse ${ }^{53}$ de Rimbaud, ou du « mundo en llamas $»^{54}$ de Panero.

Improbable remède, le dualisme entre Satan et Dieu cède la place à une indétermination, une confusion des deux. Pour mieux semer le trouble, Panero alterne les hymnes à Satan (cinq au total) et ceux à Dieu ("Himno a Dios el padre ${ }^{55}$ et «Oh Señor Jesús $»^{56}$. En outre, Pierre Brunel met en exergue le principe même du contre-évangile qui s'applique tant au Français qu'à l'Espagnol : «Il n'oppose l'Enfer et le Paradis que pour les démythifier l'un et l'autre, et d'un seul mouvement »" ${ }^{57}$. Le titre du poème «Adorar a Dios es odiar a los hombres $»^{58}$ illustre cette mise au pilori :

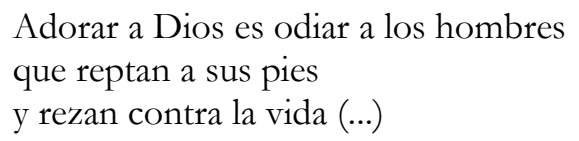

Enfin, remémorons-nous la réécriture panérienne des «Litanies de Satan ${ }^{59}$ de Baudelaire dans l'« Himno a Satán ${ }^{60}$ où le locuteur procède à une véritable inversion des rôles, désignant Satan comme protecteur charitable (tú ayudas a los débiles / mejor que los cristianos) et les enfers comme un lieu de vie et de création poétique, tandis que la terre et le royaume de Dieu renferment misère et mort (y odias esta tierra / donde moribundos descalzos / se dan la mano día tras día / buscando entre la mierda / la razón de su vida). D'autre part, Panero et Rimbaud

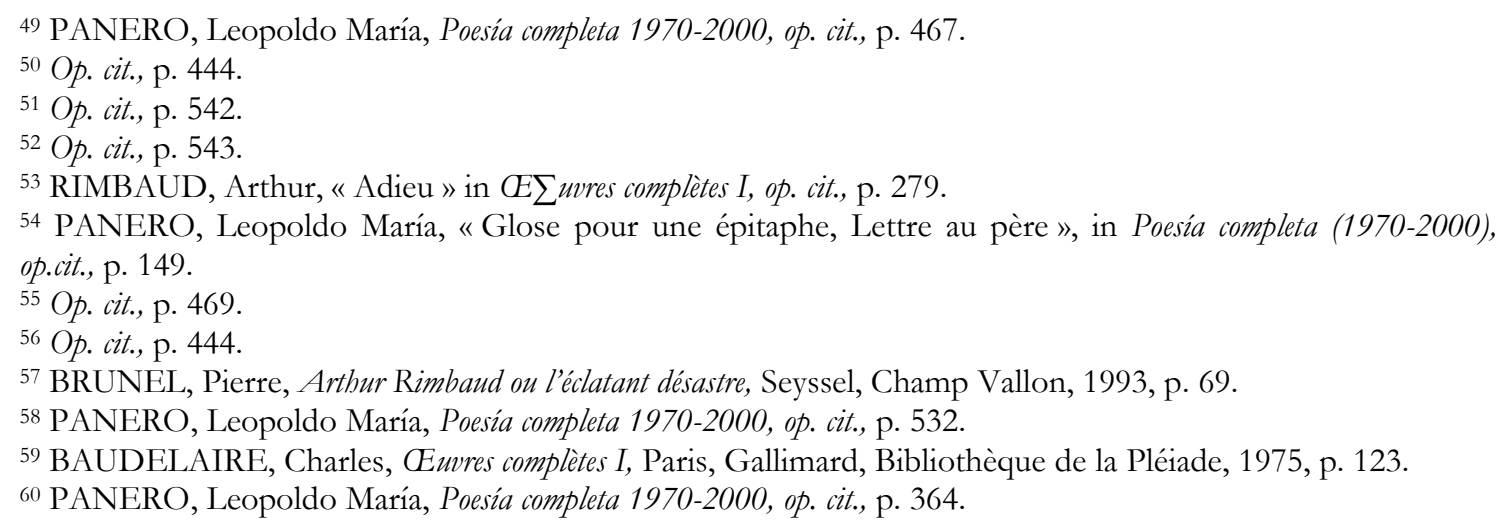


construisent leur contre-évangile sur une intertextualité biblique extrêmement dense. Par exemple, Rimbaud dans «Matin » ${ }^{61}$ réécrit l'épisode des rois mages, tout comme l'« Himno a Satán ${ }^{62}$ de Panero :

Du même désert, à la même nuit, toujours mes yeux las se réveillent à l'étoile d'argent, toujours, sans que s'émeuvent les Rois de la vie, les trois mages, le cœur, l'âme et l'esprit.

(...) rociaremos con vino, orina y

Sangre las iglesias

regalo de los magos (...).

Les deux poètes usent du substantif «mages » qui démythifie les figures bibliques en leur ôtant leur condition royale. Au trinôme originel de l'encens, de l'or et de la myrrhe se substitue le trinôme blasphématoire de Panero et le trinôme païen de Rimbaud. Au final, la dystopie de Rimbaud et de Panero aboutit au chaos. L'échec de la quête du contre-Idéal signe la fin des fantasmes. Le poète, suite à cette expérience, capitule devant les tensions les plus insolubles de sa vie spirituelle, ne trouvant pas l'inconnu qu'il cherche, il se tait devant ce monde qu'il a lui-même fait voler en éclat.

Néanmoins, le culte de la transgression et de la critique ne s'interrompt pas après cet échec. Rimbaud et Panero poursuivent la quête d'un renouveau discordant et déformant à travers les deux principes majeurs qui n'ont cessé de questionner l'œuvre rimbaldienne : la «monstruosité » et le " dérèglement». La première lecture produit toujours le même effet de heurt et de malaise. Cependant, la «monstruosité » n'intervient pas à titre gratuit, elle travaille à ce " dérèglement des sens ${ }^{63}$ que Rimbaud réclame très tôt comme pilier de son art poétique. Il s'agit de perturber l'appréhension du poème en convoquant tous les sens dans un tourbillon anarchique. Très vite, l'anomalie qui suscitait à première vue le rejet, attire l'attention et fait valoir la singularité du travail de création. L'étrangeté n'altère le monde que pour mieux le dépeindre lucidement dans toute son imprécision.

Tout d'abord, le vice et la violence se propagent dans les œuvres rimbaldienne et panérienne par le biais de quatre ressorts essentiels : le blasphème, la scatologie, la sexualité, la mort. Lina Iglesias nuance toutefois : «L'œuvre de Panero, même si elle porte en elle les traces d'une sorte de désastre, forme un ensemble cohérent, construit sur un discours où se mêlent violence langagière et intensité lyrique $»^{64}$. Cette dynamique iconoclaste et pervertissante fonctionne donc comme un moteur de la création poétique. Túa Blesa nomme cette poésie de la monstruosité, la « parade des monstres ${ }^{65}$ où défilent vampires, vieilles et vieux, moribonds, diables et autres cadavres. Pierre Brunel parle, lui, d'un « éclatant désastre » ${ }^{66}$ reprenant les mots de Rimbaud.

L'homosexualité est l'un des temas candentes de l'univers panérien que le poète revendique ouvertement. Il pousse l'abjection à la limite de la nécrophilie et de la

\footnotetext{
${ }^{61}$ RIMBAUD, Arthur, CEuvres complètes I, op. cit., p. 277.

62 PANERO, Leopoldo María, Poesía completa 1970-2000, op. cit., p. 364.

${ }^{63}$ RIMBAUD, Arthur, Euvres complètes I, op. cit., p. 340.

${ }^{64}$ IGLESIAS, Lina, «Figures de l'enfant et de la mort dans l'œuvre poétique de Leopoldo María Panero », in Figures de la mort dans la littérature de langue espagnole, (éd. Amadeo López), Université de Paris X-Nanterre, GRELPP, CRIIA, 2006, p. 81- 94.

65 BLESA, Túa, Leopoldo María Panero, el último poeta, Madrid, Valdemar El Club Diogenes, série «Autores Españoles », 1995. Notre traduction.

${ }^{66}$ BRUNEL, Pierre, Arthur Rimbaud ou l'éclatant désastre, Seyssel, Champ Vallon, 1993, p. 195.
} 
pédophilie en représentant l'acte sexuel avec un petit garçon ("Himno a Dios el padre ») ${ }^{67}$ ou avec un cadavre ("Cópula con un cuerpo muerto ») ${ }^{68}$. La manipulation du scatologique par Panero relève presque d'une constante dans son œuvre, ce qui en fait l'héritier jusqu'auboutiste du poète Français. Entre tous, "En mis manos acojo los excrementos » ${ }^{69}$ se fait particulièrement explicite: En mis manos acojo los excrementos / formando con ellos poemas. Tout comme "'La destruction fut ma Béatrice'»" ${ }^{70} \mathrm{Ob}$ vida, dime (...) / qué sendero hay que no condurca al excremento.

Túa Blesa explicite le cheminement du poète : «Le discours poétique émane du sujet comme ses déjections. (...) L'excrément c'est le poème : l'œuvre arrachée ${ }^{71}$. Par conséquent, la monstruosité n'est pas uniquement à considérer au premier degré, elle se lit également au moyen de la destruction du réel. La dissolution s'opère à travers la corruption des choses. Le beau et le laid, nous l'avons vu, ne sont plus des concepts normatifs et opposés, mais des contradictions qui engendrent une dynamique des contrastes, essence du poème. L'héritage-Baudelaire affleure avec la référence explicite au poème «Les sept vieillards $»^{72}$ et son écho panérien, les « Poemas de la vieja » ${ }^{73}$.
Me dijo mi amor un día
« sólo sabes ladrar, vieja
ni el demonio en el aire inmundo
es peor que tu figura
que tu pellejo grasiento
que ladra aún sobre el poema».

Au final, pour Panero, «La littérature est - ou doit être - une critique de la réalité, y compris lorsque pour l'être elle doit s'éloigner de celle-ci, (...) rendant la lecture difficile ou impossible, comme chez Gongora et Mallarmé $»^{74}$. Si la monstruosité sert la critique, c'est au moyen de l'altération des sens et de la raison. En outre, la transgression orchestrée par les deux poètes s'exprime fondamentalement à travers une écriture de l'erreur, des «couacs », de la "subversification », du dérèglement de la forme. Comme le rappelle Túa Blesa, "pour contenir le flux de son discours poétique, Panero adopte majoritairement le vers libre, informel, typique de cette longue tradition initiée par Rimbaud ${ }^{75}$. Il s'agit de défaire le concept traditionnel du poème à travers une subversion lyrique qui illustre une subversion de la vie. Il laisse toutefois encore la place aux vers classiques - en majorité l'alejandrino et l'octosyllabe. Héritière de la Modernité la poésie panérienne laisse transparaître une insoumission à un code langagier et social, insoumission qui finit par nier la poésie elle-même dans la mesure où elle ne laisse pas toujours le sens advenir. La «subversification» se module autour d'une ponctuation chaotique (insertion de signes, d'alphabets étrangers, de smileys, parenthèses ouvertes mais jamais refermées, majuscules intempestives), parfois même inexistante, qui neutralise la diction classique et dérègle le discours et la typographie : le corps du poème se trouve corrompu, implosant littéralement,

\footnotetext{
${ }^{67}$ PANERO, Leopoldo María, Poesía completa 1970-2000, op. cit., p. 469.

${ }^{68}$ Op. cit., p. 166.

${ }^{69}$ Op. cit., p. 392.

70 Op. cit., p. 482.

${ }^{71}$ BLESA, Túa, Leopoldo María Panero, el último poeta, op.cit., p. 115. Notre traduction.

72 BAUDELAIRE, Charles, CEuvres complètes I, op.cit., p. 87.

73 PANERO, Leopoldo María, Poesía completa 1970-2000, op. cit., p. 547-550.

74 PANERO, Leopoldo María, «Última poesía no-española », Poesía, n²4, Mallorca, juin 1979, p. 110.

75 BLESA, Túa, Leopoldo María Panero, el último poeta, op.cit., p. 23. Notre traduction.
} 
et le lecteur, déstabilisé par l'absence de repères. Pour le plaisir, observons un exemple extrême, extrait du poème $« 6 »^{76}$ :

Vinum Sabbati (espada

destruye a copa)

Seth, el comedor de muertos

luz, esfera

gotear impasible de los cirios

(SIPHER, SEPHORA

sobre el altar vacío

SWORD, AUTUMN

espada sobre el otoño

SÓLO

AQUELLO QUE NO EXISTE

NO PUEDE MORIR

que frágilmente enciende

(y una absurda pregunta

las desiertas avenidas:

Elena, José Sainz, y Eduardo y Ana

y Heli de los labios inmóviles, V.O. finalmente

tardía aurora parecida a un estertor

(they all go)

cuchillada final sobre abril moribundo

into the darkness

(they all go

$[\cdots]$

y el amor

Jean-Pierre Richard condense ce que pourrait être l'art poétique panérien: «Un dérèglement comme règle de vie, une anarchie pour discipline, une ignorance pour étude (...) car s'il veut la folie, c'est par raison; s'il se lie au hasard, c'est en vertu d'un plan très sûr ; l'incontrôlé même lui est système $»^{77}$. Ainsi, Rimbaud et Panero élaborent une poétique féconde du chaos. En effet, pour changer le monde, il faut ramener le Chaos, prélude nécessaire d'une nouvelle Genèse.

\section{Au-delà de la liquidation, un possible renouveau poétique}

Ainsi, par-delà la liquidation perce pourtant l'intuition d'un idéal poétique à reconstruire, à prolonger; ou plutôt de ce fameux «contre-idéal» à poursuivre. L'extrémisme des postulats formels et thématiques de Panero et la constante exacerbation de la finitude et de la caducité semblent soudainement préfigurer la création. En effet, paradoxalement, la refonte du poème sous le poids des références culturelles sous-tend potentiellement une poésie inédite, dont la mutation reste inachevée.

D'ordinaire, l'abondance catalyse le néant parce qu'elle sature l'objet-poème jusqu'à son implosion polymorphique et polysémique. La multiplication des insertions culturelles et

76 PANERO, Leopoldo María, Poesía completa (1970-2000), op.cit., p. 94.

${ }^{77}$ RICHARD, Jean-Pierre, Poésie et Profondeur, Paris, Points Seuil, 1955, p. 299. 
idiomatiques déforme et fragmente l'œuvre panérienne. Mais, au final, comment mieux représenter l'expérience contemporaine ? Le prisme kaléidoscopique de Panero correspond à la nécessité de parvenir à déterminer un langage capable d'évoquer l'univers de la modernité et l'évolution chaotique d'un monde caduc. Ainsi, pour que « réalisme il y ait ${ }^{78}$, la parole doit être plurale et ambivalente au risque d'être dérangeante. La forme doit suggérer l'expérience du vertige, l'angoisse de la dégradation, la réaction face à des phénomènes de destruction latents. Voyons ainsi, le poème "Ash Wednesday » ${ }^{79}$ qui reprend avec ce titre en anglais dans le texte, un poème éponyme de T. S Eliot tout en incluant, à la fin et en français dans le texte, un vers de Mallarmé. Et, au beau milieu de ce court poème, l'insertion du substantif allemand « ungrund » qui représente le néant dans la philosophie de Maître Eckart, théoricien de la mystique rhénane :

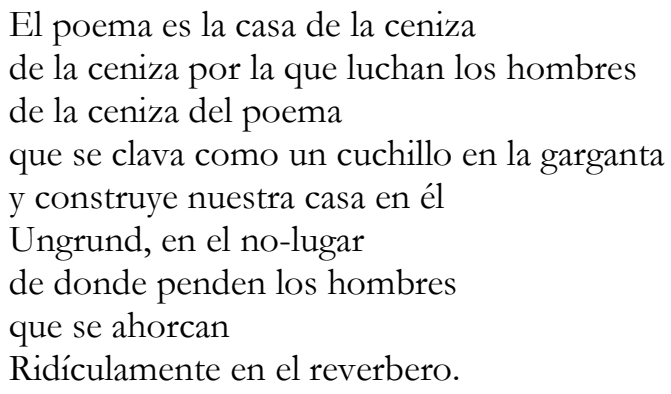

(Mallarmé : « vont se pendre ridiculement au réverbère »)

Joint au système d'échos et de répétitions qui structure le poème tout en miroirs et en parallèles, le trinôme idiomatique et littéraire densifie encore le réseau de significations. Or, derrière ce système clos, circulaire et martelant, il y a une forte cohérence interne. L'élément central qu'est le néant sert à la fois le discours méta-poétique et le discours critique. Au travers de l'analogie entre la «maison» et le "poème», réunis autour du substantif dysphorique "cendre », il définit à la fois le monde et la poésie au sein desquels le locuteur erre, prisonnier moribond. En effet, au « ungrund » qui symbolise une forme de néant ténébreux, se superposent le poème d'Eliot qui scande «je n'espère plus » et le funeste vers mallarméen. Panero met en scène l'enfermement de l'individu et du poète, condamnés par leur condition humaine et donne à voir ce même cercle vicieux dans la poésie. Car la poésie doit être reflet, porte-voix et victime à son tour de cette finitude.

Panero touche alors presque au décadentisme : sa poésie, comme celles des poètes de la Modernité, entend dénoncer l'environnement dans lequel elle évolue. Manifestation d'une dégénérescence, d'une perte d'estime pour la grandeur nationale - et l'Humanité, dans une acception plus globale -, son œuvre veut traduire les émotions propres aux époques de déclin. Esthétisme de la dégradation, de la décomposition, il représente la névrose des esthètes qui s'isolent du monde contemporain. Adoré Floupette dit qu'il s'agit de traduire « le détraquement exquis de l'âme moderne » par la « névrose de la langue »" Et le décadentisme n'est pas sans manifester un goût pour la provocation, la transgression, les artifices, mais surtout pour une remise en question de la normalité. Pensons encore à «Ann

\footnotetext{
${ }^{78}$ CARNERO, Guillermo, "Puisque réalisme il y a », en français dans le texte in Ensayo de una teoría de la visión, Madrid, Hiperion, 1983, p. 177. Référence aussi au chapitre VII de l'appendice de L'art Romantique de Charles Baudelaire.

${ }^{79}$ PANERO, Leopoldo María, Poesía completa 1970-2000, op. cit., p. 561.

${ }^{80}$ FLOUPETTE, Adoré, cité par GROJNOWSKI, David, Le dictionnaire du littéraire, Paris, PUF, 2004, p. 142.
} 
Donne : Undone ${ }^{81}$ où Panero reprend d'ailleurs les mots de Lautréamont en citation préliminaire, avant de céder la place à William Wilson, personnage "extraordinaire» d'Edgar Allan Poe. Rappelons-nous ce personnage confronté à son double qu'il finira par tuer, réalisant à cet instant tragique, s'être en réalité donné la mort, à lui-même.

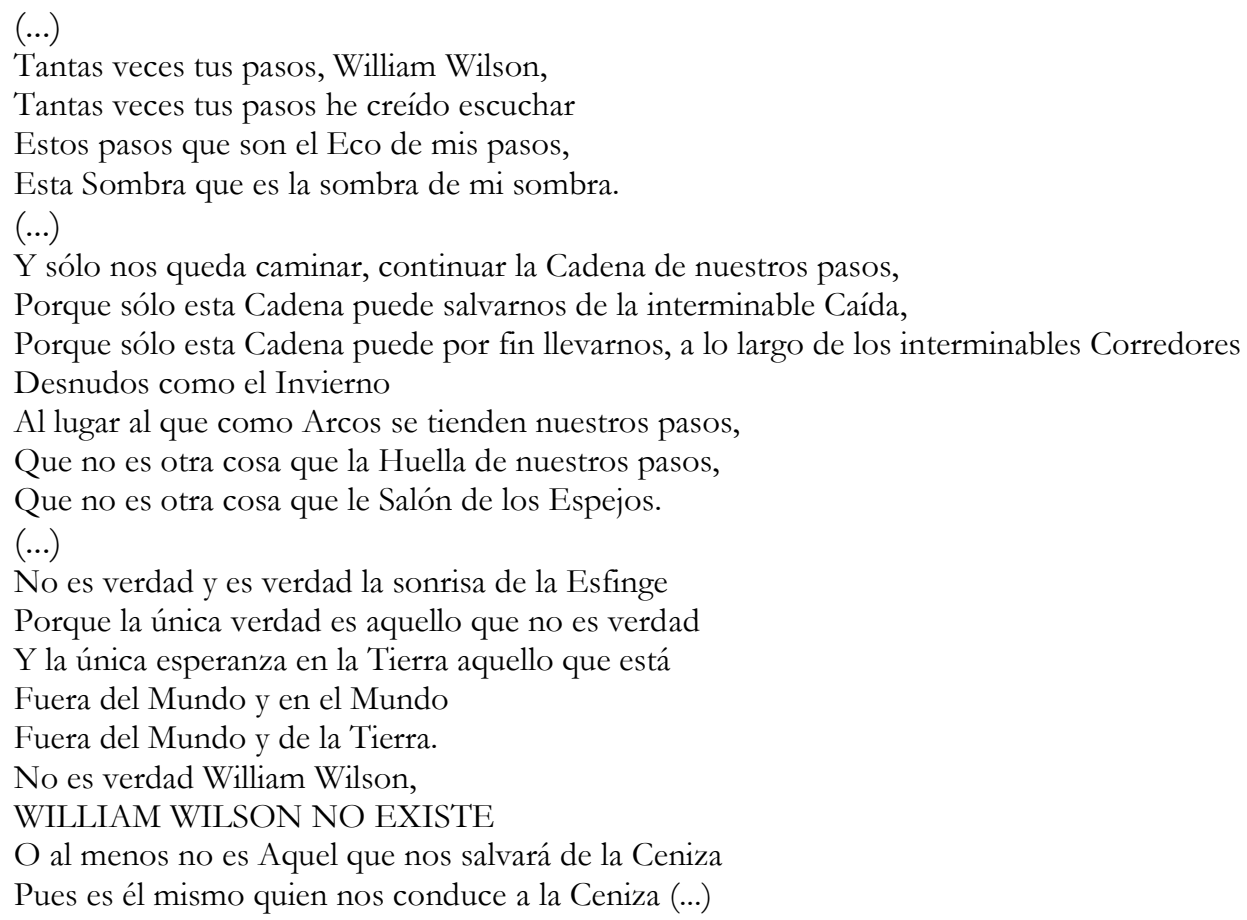

L'intertexte littéraire, auquel s'ajoute la référence symbolique à la Galerie des Glaces, épouse parfaitement la réflexion métaphysique que mène le locuteur. Le thème traditionnel de la finitude est ici transmué par la référence culturelle - référence qui est détournée et pervertie - et il revêt, tout comme la méditation métaphysique par là même, une dimension neuve. Il y a donc d'une part réécriture de l'intertexte et d'autre part, rénovation d'un poncif de la poésie : dans les deux cas, l'abondance des motifs culturels épars participe d'un renouveau poétique.

La démarche herméneutique, plus intuitive que rationnelle désormais, doit accepter de céder à la perte de repères et, conséquemment, de renoncer à sa grille de lecture traditionnelle. Révolution subversive, elle participe d'une écriture du cogito interrumptus catalysée par une folie raisonnée (cette «lucide folie ${ }^{82}$ que Marie-Claire Zimmermann a immédiatement mise en exergue) qui transcende l'essence originelle de la poésie. Et Panero, par ce choix d'une mosaïque culturelle et babélienne, façonne - parfois, souvent même, par la force - un nouvel interlocuteur. En atteste Paul Ricœur: "Un poème est un 'hasard vaincu mot par mot' (...) Comprendre le poème c'est pour le lecteur vaincre à son tour l'apparence du fortuit et retrouver non pas nécessairement par entendement, mais par accueil poétique 'l'air du chant sous le texte conduisant la divination'» ${ }^{83}$. Soit un nouveau protocole de lecture. L'art poétique panérien conçoit le poème comme sujet et objet d'une métamorphose: la poésie, l'univers et l'individu sont devenus des hybrides. Le sujet polymorphe évolue dans un contexte duel, toujours ambivalent, et le discours ne peut que

${ }^{81}$ PANERO, Leopoldo María, Poesía completa 1970-2000, op. cit., p. 71.

82 ZIMMERMANN, Marie-Claire, Poésie espagnole moderne et contemporaine, Paris, Armand Colin, 1995, p. 179.

${ }^{83}$ RICCEUR, Paul, Philosophie de la volonté, Tome I, Paris, Aubier, 1949, p. 380-381. 
s'adapter à ces contradictions qui régissent cet univers contemporain. De ce fait, si la parole se transforme en une onde polysémique et complexe, c'est à l'initiative d'un sujet à l'identité mouvante. Dépersonnalisé, celui-ci favorise la suggestion et la transcendance du sens dans la polyphonie, tronquant les attentes du lecteur, tributaire d'une forme de déchiffrement.

Désormais, le lecteur doit mériter l'accès au sens voilé par les masques et les ombres, les excès et le non conventionnel. La transgression formelle jointe à l'ambiguïté de la parole, du sujet et des objets bouscule les repères du public, malmené de surcroît par l'intertextualité et les références culturelles. Rien ne saurait arrêter cette nécessité de désarçonner le lecteur. Preuve en est faite par l'incursion d'éléments vulgaires et scatologiques mais aussi véhéments ou tabous. Brutaliser le lecteur est un acte éthique. Panero entend remettre en cause cet acteur incontournable et le pousser à se remettre en question parce qu'il est le maillon essentiel de la transmission du poème et de la propagation de ses significations. La démarche herméneutique se construit désormais sur la mise à mort des normes et des codes : l'érudition n'est plus la condition sine qua non pour comprendre son œuvre. Túa Blesa analyse ce paradoxe : «Le lecteur est appelé vers les silences, devenus écriture oubliée, il est (...) ensorcelé par le désir de sens, il doit lui-même poétiser, il doit tracer le sens $»^{84}$. Ainsi, le renouveau prend racine dans le silence, le blanc de la page, grâce à la zone franche que ce dernier ménage pour le lecteur. Serait-ce alors le but final de l'anéantissement du carcan-poème orchestré par Rimbaud et Panero ? En libérant un poème hors-normes, un «hors-poème », ils exhortent le lecteur à participer à sa reconquête. Le lecteur s'engage à reconstruire le sens et la parole, à donner à entendre et à voir un "contre-poème». Panero, incapable de se soustraire à la fin qui le dévore et l'asservit, dévoile sa stratégie : faire du blanc et du silence le lieu d'un prolongement du poème par le lecteur. Et de conclure : ven aqui, he / construido este poema como un anzuelo / para que el lector caiga en ép ${ }^{55}$.

Au final, alors même que sa poésie s'articule autour d'un projet apocalyptique, accroissant d'une part, sa déconstruction formelle par le vide et l'entremêlement des langues, et d'autre part, sa destruction thématique par le blasphème, l'hétérogénéité des références et la finitude, il semblerait finalement que Panero travaille non pas à une liquidation du poème mais à sa régénération. L'expérience constante des limites, tient moins de la provocation stérilisante qu'il n'y parait, et s'achemine vers une transcendance du signifié s'ouvrant sur l'inachèvement du sens. Cette suspension de l'aboutissement du poème tend alors vers une expansion exponentielle de celui-ci, vers son débordement, mû par le miroitement des œuvres dans l'CEuvre. En convoquant plusieurs idiomes, divers héritages culturels et des niveaux de connaissances distincts, Panero compose une dystopie aux portes de l'universel, qui n'a finalement rien à envier au songe de Babel.

Parallèlement, cette intertextualité dévoile, par-delà le mode d'écriture, un mode de lecture: en effet, l'écriture référentielle figure une infinie dynamique de relecture chez Panero. Le poète se révèle ainsi en lecteur originel et sa poésie, Bibliothèque onirique de l'Europe, se réalise dans cette propagation toute en échos et en reflets de la Poésie pure. Ainsi, entreprend-il de ciseler à coup de hache une poésie désormais taillée pour un contrelecteur.

${ }^{84}$ BLESA, Túa, Leopoldo María Panero, el último poeta, op.cit., p. 34. Notre traduction.

85 PANERO, Leopoldo María, Poesía completa 1970-2000, op. cit., p. 544. 